\title{
DIGITAL TRANSFORMATION OF AIR COMPRESSOR UTILIZATION BASED ON AI, ML IN SMART FACTORY MANAGEMENT
}

\author{
MILAN KUMAR ${ }^{1}$, V. M. SHENBAGARAMAN ${ }^{2}$ \& ANKUSH GHOSH \\ ${ }^{1,2}$ SRM Institute of Science and Technology, Chennai, India \\ ${ }^{3}$ The Neotia University, West Bengal, India
}

\begin{abstract}
Digital transformation is not almost disruption or generation. It is approximately cost, people, optimization and the functionality to rapidly adapt whilst such is needed via a wise use of technology and facts. It leverages technology to make charge for numerous stakeholders, innovate and gather the skills to rapidly adapt to changing conditions. With the extensive deployment of sensors, IOT and Artificial Intelligence there's a growing need of handling big manufacturing data characterized by high velocity, high variety, and high volume. Machine learning provides advanced analytics tools for processing and analyzing large manufacturing data. It usually presents a comprehensive survey of commonly used deep learning algorithms and discusses their applications toward making manufacturing "smart". The development of artificial intelligence with machine learning technologies and their benefits are firstly discussed. Subsequently, computational methods supported machine learning are presented specially aim to improve system performance in manufacturing. Several parameters of smart manufacturing have been analyzed using deep learning models and corresponding results have been discussed. At the end, the emerging things of research on predictive data analysis of a smart manufacturing system using machine learning is highlighted, and future trends and challenges associated with deep learning for smart manufacturing are summarized.

KEYWORDS: Smart Manufacturing, Digital Transformation, Artificial Intelligence, Machine Learning
\end{abstract}

Received: Jun 06, 2020; Accepted: Jun 26, 2020; Published: Aug 25, 2020; Paper Id.: IJMPERDJUN2020877

\section{INTRODUCTION}

Even as Digital transformation is predominantly carried out in a enterprise context, it impacts specific companies like governments, public area companies and groups which might be concerned in tackling societal challenges which include pollutants and getting older populations via leveraging one or extra of these present and rising technologies. Artifical Intelligence will not just be about automation or predictions. The wide variety of precise use instances will growth at lightning velocity and complicated algorithms are going to be required to execute these obligations. self sustaining vehicle becomes a mainstream reality. Self-programming techniques have already end up powerful sufficient to shape AI broaden its personal code; supplying gigantic possibilities to programmers for constructing unattainable structures. The increased optimization and velocity of improvement in artificial Intelligence has caused its adoption in various industries at breakneck velocity, along with hello-tech, telecom, automobile, monetary carrier and healthcare, to call some. This year AI is expected to automate more complex strategies, providing ahead-looking intelligence and creating commercial enterprise value. As cellular becomes even extra powerful with 5G, there'll be ripple results for the duration of the producing enterprise [1-4]. There can be advanced connectivity for robotics and automation in the manufacturing facility and quicker real-time analytics from field operations.

In a Smart factory a manufacturing facility bodily manufacturing processes and operations are blended 
with virtual era, smart computing and massive records to create a greater opportunistic gadget for groups that target manufacturing and deliver chain control [5-6]. The Smart factory unit are an thing of enterprise 4.0, a brand new section inside the business Revolution that focuses closely on actual-time statistics, embedded sensors, connectivity, automation, and machine studying. As factories evolve in mild of the information revolution, corporations need to reconsider how they manage the whole thing from automation strategies to team of workers development tactics. Smart manufacturing plays a significant role in the energy consumption [7-9]. Along the way, manufacturers will need modernized gear, including robust, flexible employer aid planning systems as a statistics and transactional spine, that help them adapt quickly as they construct toward a smart-factory future.

The main objective of this work is to is to identify the opportunity to improve energy efficiency of all the compressor out of the six compressors and save the extra power utilized by compressors in theshopfloor.

\section{INTELLIGENT DATA ANALYSIS ON AIR COMPRESSOR UTILIZATION}

Data-driven intelligence models model complex multivariate relationships between data, requiring no in-depth understanding of the physical behavior of the system. Data driven intelligence has attracted extensive research effort for manufacturing data distilling and decision making $[9,10]$. In data mining techniques are classified into five categories, including characterization and description, association, classification, prediction, clustering and evolution analysis. The barriers to data-driven deciding in manufacturing also are identified. Typical machine learning techniques are reviewed in for intelligent manufacturing, and their strengths and weaknesses are also discussed in a wide range of manufacturing applications. A comparative study of machine learning algorithms including Artificial Neural Network, Support Vector Machine, and Random Forest is performed for machining tool wear prediction. The schemes, techniques and paradigm of developing decision making support systems are reviewed for the monitoring of machining operations, and these techniques include neural networks, fuzzy logic, genetic algorithms, and hybrid systems. The potential benefit and successful application examples of typical machining learning techniques including Bayesian Networks, instance-based learning, Artificial Neural Network, and ensemble methods are discussed in. Cloud enabled prognosis techniques including data driven approach, physics based as well as model-based techniques are reviewed in, with the benefits from both advanced computing capability and information sharing for intelligent decision making. Traditional machine learning is usually designed with shallow structures, such as Artificial Neural Network, Support Vector Machine, and logistic regression, etc. By coping with limited handcrafted features, it achieves decent performance in a variety of applications. However, the massive data in smart manufacturing imposes a variety of challenges, such as the proliferation of multimodal data, high dimensionality of feature space, and multicollinearity among data measurements. These challenges render traditional algorithms struggling and thus greatly impede their performance.

As a breakthrough in artificial intelligence, deep learning demonstrates outstanding performance in various applications of speech recognition, image recondition, natural language processing, multimodal image-text, and games $[11,12]$. Deep learning allows automatically processing of data towards highly nonlinear and complex feature abstraction via a cascade of multiple layers, instead of handcrafting the optimum feature representation of data with domain knowledge [13]. With automatic feature learning and high-volume modelling capabilities, deep learning provides a complicated analytics tool for smart manufacturing within the big data era [14]. It uses a cascade of layers of nonlinear processing to learn the representations of data corresponding to different levels of abstraction. The hidden patterns 
underneath one another are then identified and predicted through end-to-end optimization. Deep learning offers great potential to spice up data-driven manufacturing applications, especially within the big data era.

The data has been analyzed by correlating energy, pressure and loading/unloading patterns to understand compressors efficiency.

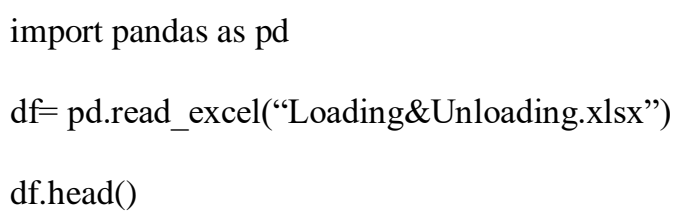

Table 1

\begin{tabular}{|c|c|c|c|c|c|}
\hline Timestamp & $\begin{array}{c}\text { Pressure P1 } \\
\text { [bar] }\end{array}$ & $\begin{array}{c}\text { Pressure P3 } \\
\text { [bar] }\end{array}$ & $\begin{array}{c}\text { Compressor1 } \\
\text { [load/unload] }\end{array}$ & $\begin{array}{c}\text { Compressor2 } \\
\text { [load/unload] }\end{array}$ \\
\hline $2020 / 02 / 2700: 00: 05$ & 10.5494566 & 10.21063805 & 1 & 1 \\
\hline $2020 / 02 / 2700: 00: 10$ & 10.5494566 & 10.21063805 & 1 & 1 & Pressure data and \\
\hline $2020 / 02 / 2700: 00: 15$ & 10.5494566 & 10.21063805 & 0 & 1 & compressorLoad/Unlo \\
\hline $2020 / 02 / 2700: 00: 20$ & 10.5494566 & 10.21063805 & 0 & 1 \\
\hline $2020 / 02 / 2700: 00: 25$ & 10.5494566 & 10.21063805 & 0 & 1
\end{tabular}

import pandas as pd

df= pd.read_excel(“Consumption.xlsx”)

df.head()

\section{Table 2}

\begin{tabular}{||c|c|c||l|}
\hline Timestamp & $\begin{array}{c}\text { Compressor1 } \\
\text { [kW] }\end{array}$ & $\begin{array}{c}\text { Compressor2 } \\
\text { [kW] }\end{array}$ & CompressorEnergyCons \\
\hline 2/23/2020 5:30 & 0.05 & 0.00 \\
\hline 2/23/2020 5:31 & 0.05 & 0.00 \\
\hline $2 / 23 / 20205: 32$ & 0.05 & 0.00 & umption data \\
\hline
\end{tabular}

\section{Energy Analytics Approach}

- Step 1: Energy consumption trend lines.

- Step 2: Power Pattern analysis.

- Step 3: Power range analysis.

- Step 4: Energy consumption hourly based on high and least hour.

- Step 5: Correlation between pressure, power and loading/unloading pattern.

- Step 6: Loading and unloading counts per day.

- Step 7: Switch analysis of load and count.

- Step 8: Switching pattern distribution 
Energy Consumption per Day

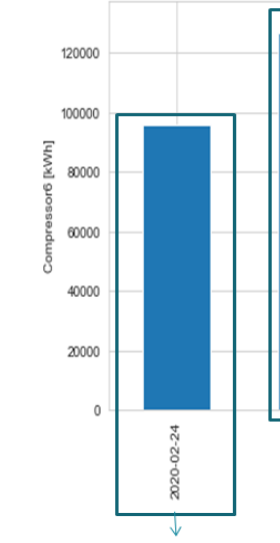

Low energy consumption cluster

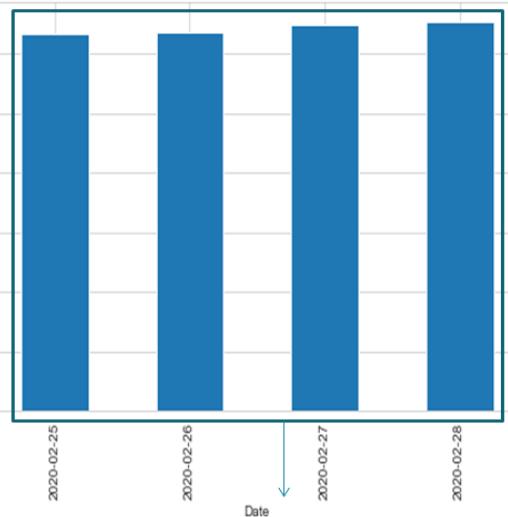

High energy onsumption cluster

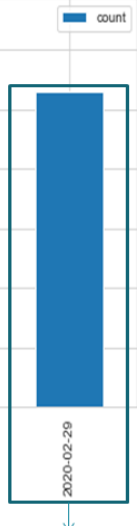

Low energy consumption cluster

Figure 1: Shows the Energy Comsumption per Day of Air Compressor Date-Wise also The Energy Pattern Whether it is Low or High is Clustered and Shown in the Graph.

Power Profile for Day 1 and Day 6 (Low Consumption Days)

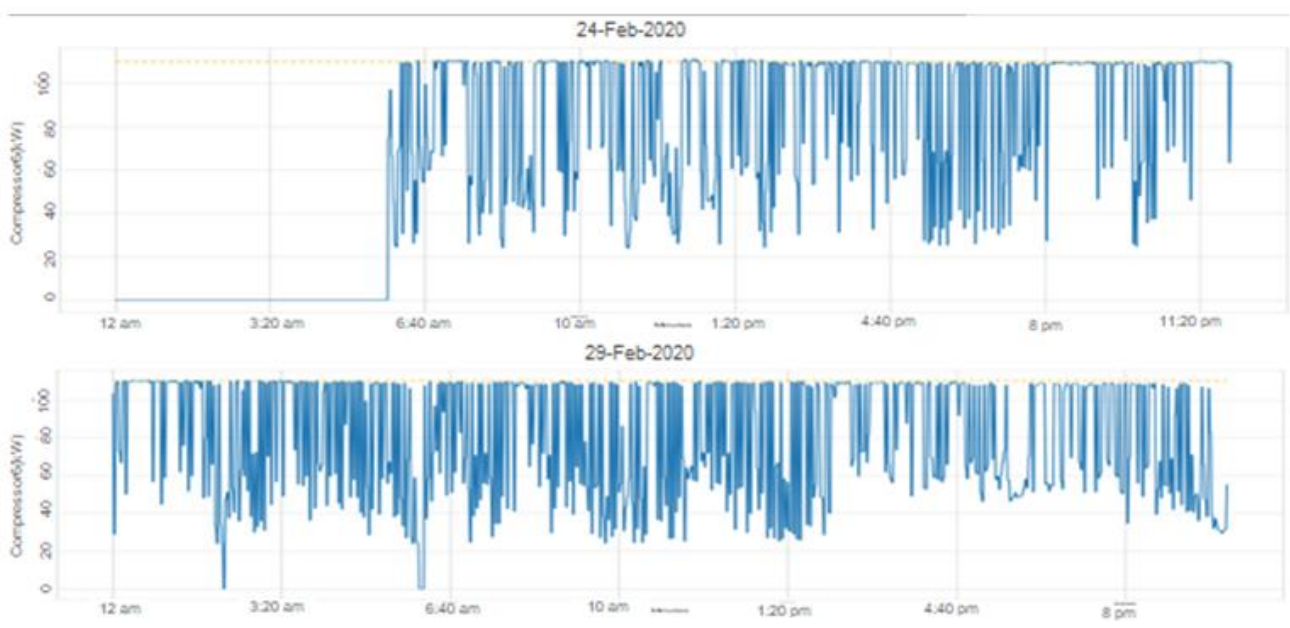

Figure 2: Shows the Power Profile of the Low Consumption Days along with Utilization at Certain Time.

\section{Power Range Plot (Day 1)}

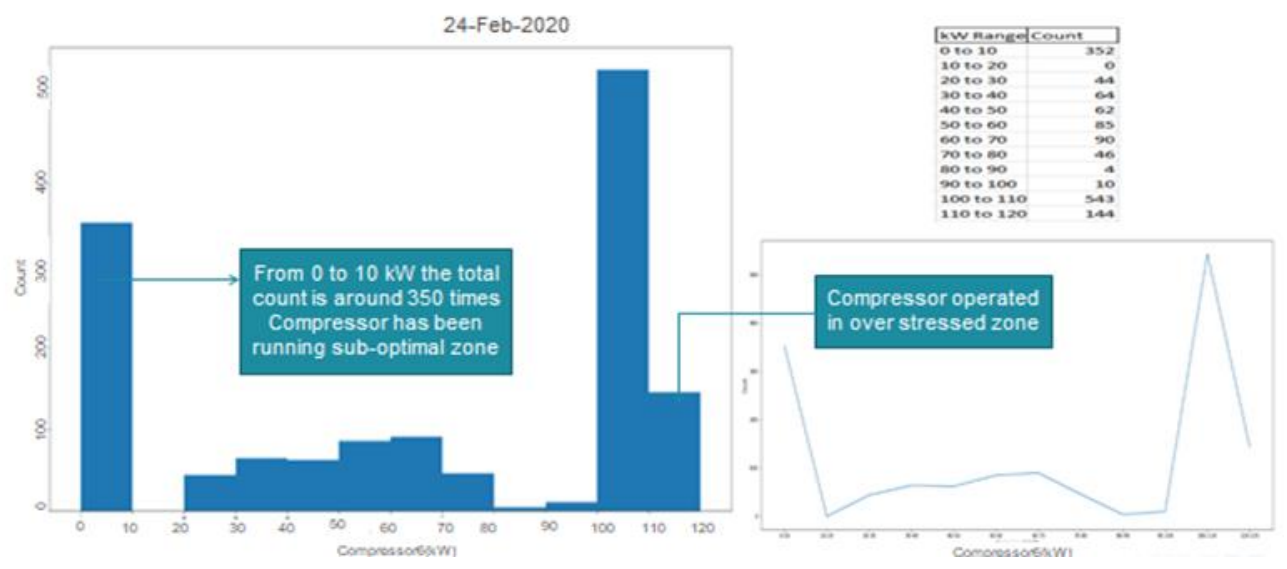

Figure 3: Shows the Count on the $\mathrm{x}$-Axis and Compressor $\mathrm{kW}$ on $\mathrm{y}$-Axis which Shows When the Compressor is Running at Sub-Optimal Zone and When it is Running in Over Stressed Zone for Day 1. 
Power Range Plot (Day 5)

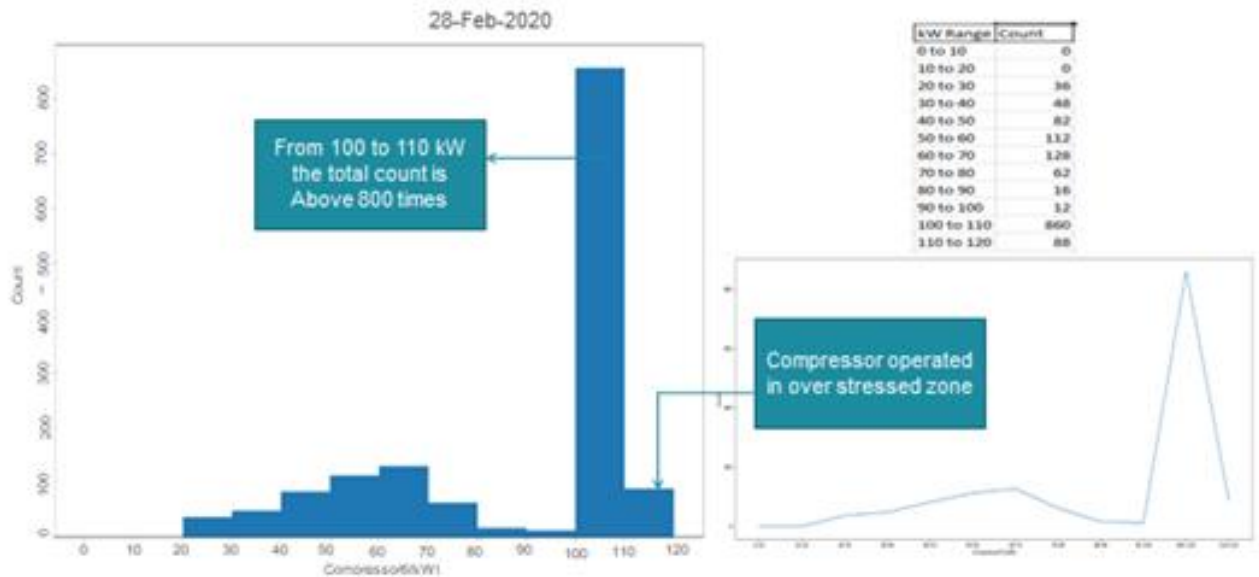

Figure 4: Shows the Count on the $\mathrm{x}$-Axis and Compressor $\mathrm{kW}$ on $\mathrm{y}$-Axis which Shows When the Compressor is Running at Sub-Optimal Zone and When it is Running in Over Stressed Zone for Day 5.

Hourly Energy Consumption

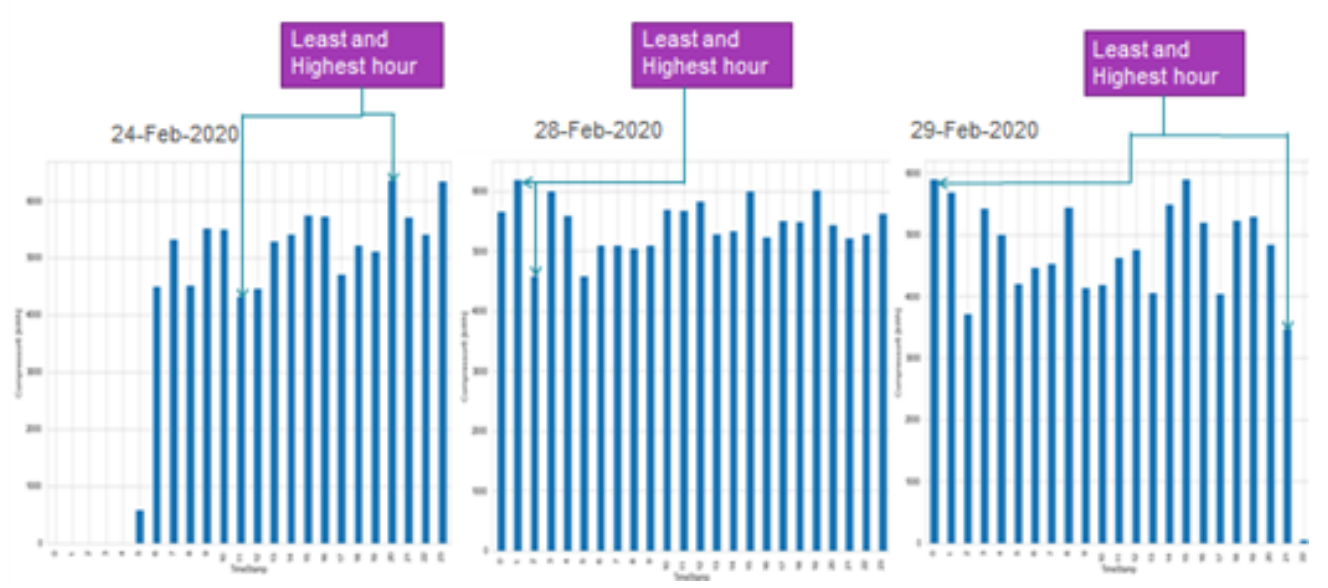

Figure 5: Shows the Hourly Energy Consumption, Least and Highest Our of Energy Consumption can be Seen.

Pressure Vs Power Vs Load/Unload (Day 1) High Consumption Hour

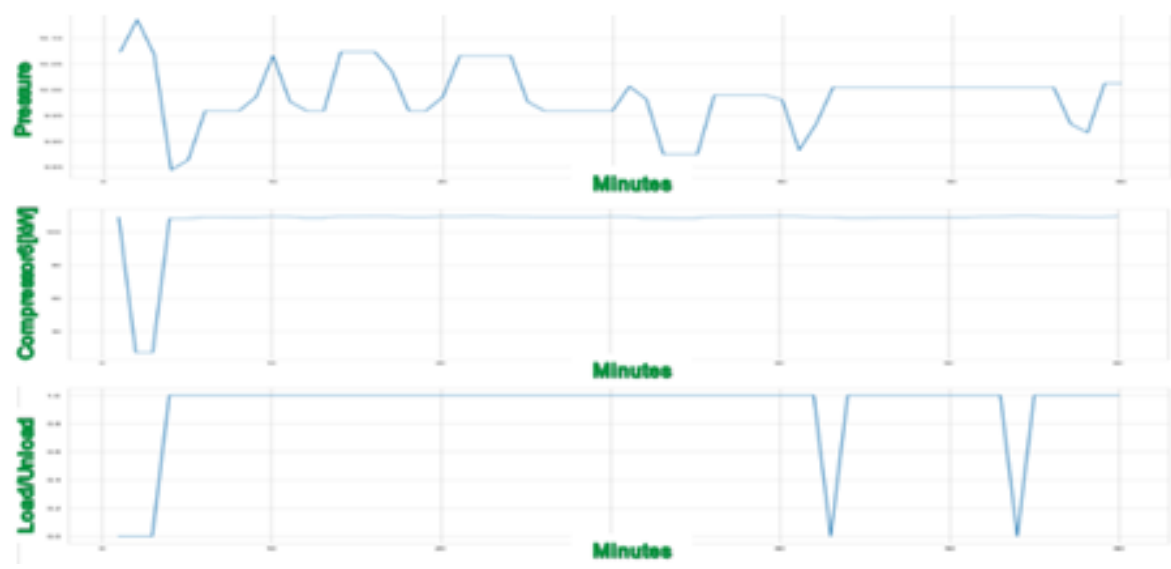

Figure 6: Shows the Graph of Pressure vs Power vs Load or Unload and High Consumption hours for Day 1. 
Pressure Vs Power Vs Load/Unload (Day 1) Least Consumption Hour

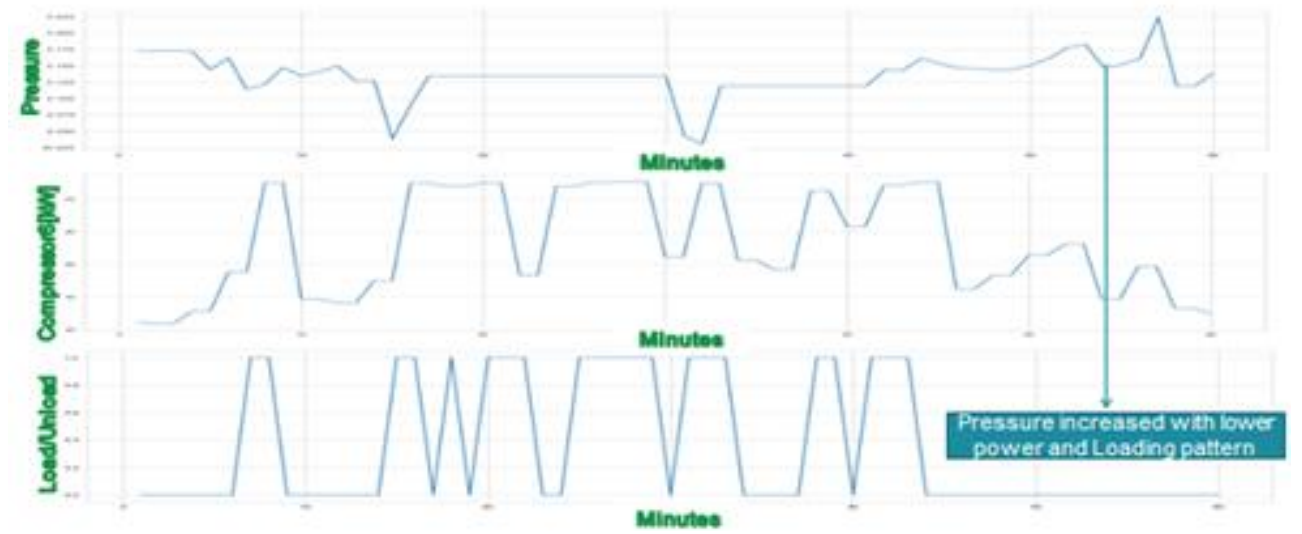

Figure 7: Shows the Graph of Pressure vs Power vs Load or Unload and Least Consumption Hours for Day 1.

Pressure Vs Power Vs Load/Unload (Day 5) Highest Consumption Hour

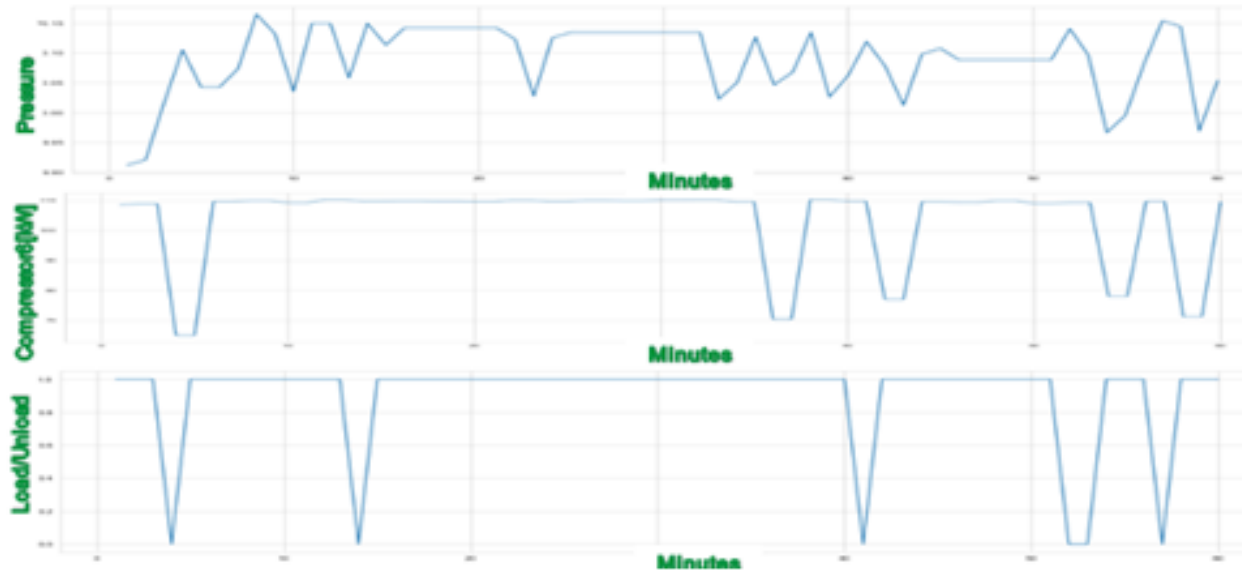

Figure 8: Shows the Graph of Pressure vs Power vs Load or Unload and High Consumption Hours for Day 5.

Pressure Vs Power Vs Load/Unload (Day 5) Least Consumption Hour

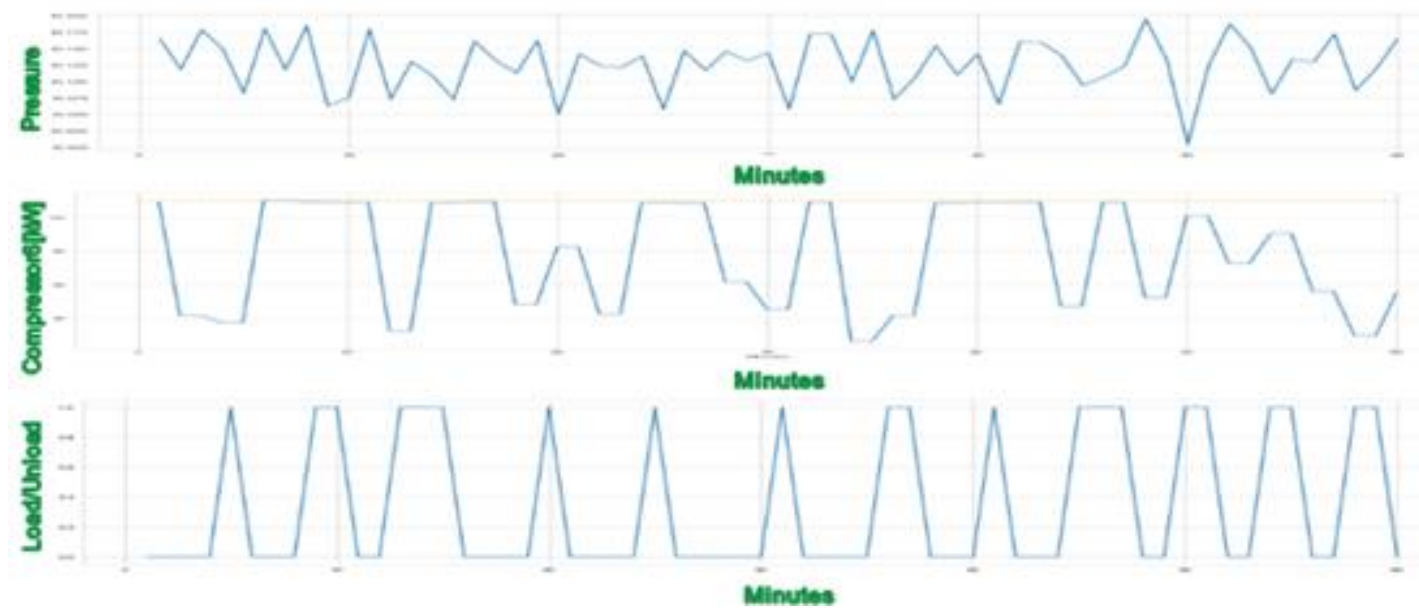

Figure 9: Shows the Graph of Pressure vs Power vs Load or Unload and Least Consumption Hours for Day 5. 


\section{Load/Unload Count per Day}

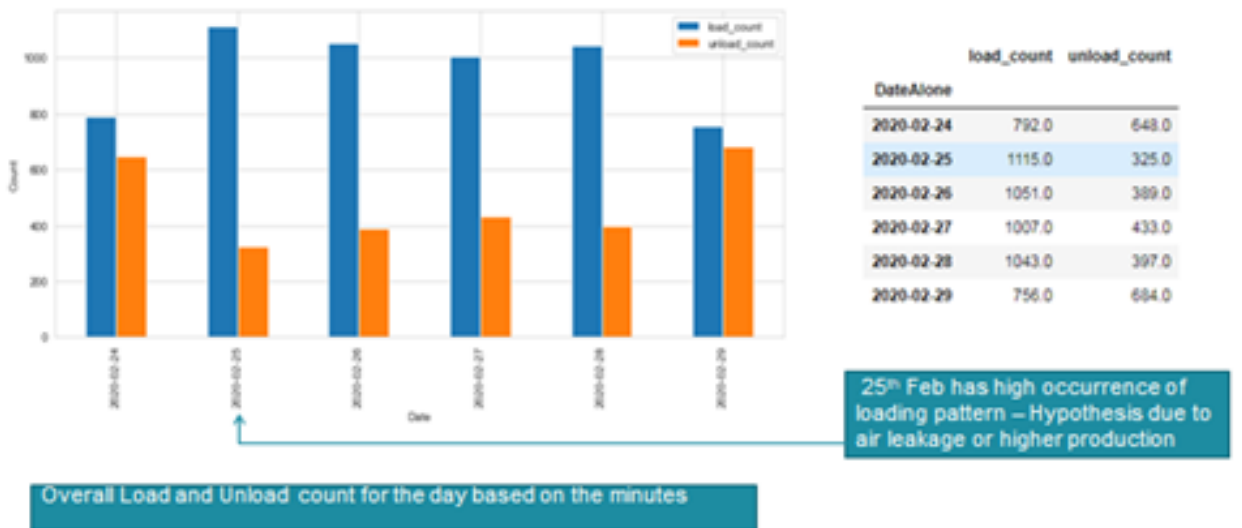

Figure 10: Shows Over All Load and Unload Count for the Day Based on the Minutes.

Switch Analysis of Load/Unload

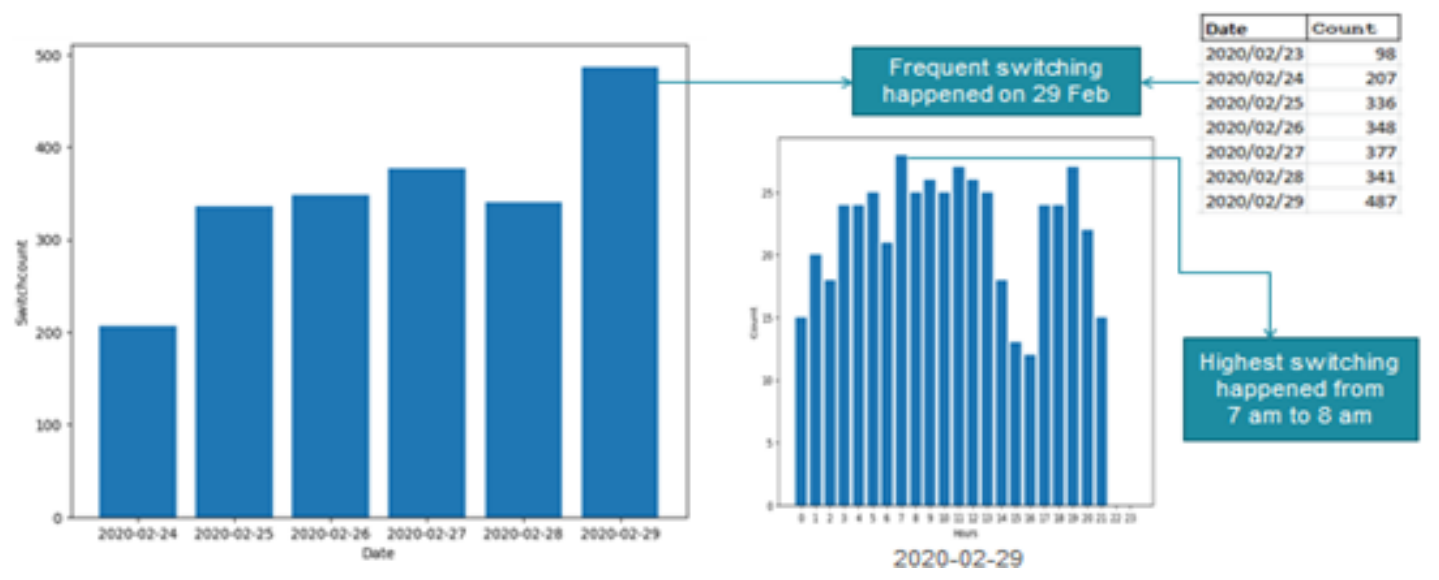

Figure 11: Shows the Analysis of Load and Unload.

Pressure Vs Power Vs Load/Unload (Day 6) Switch Count

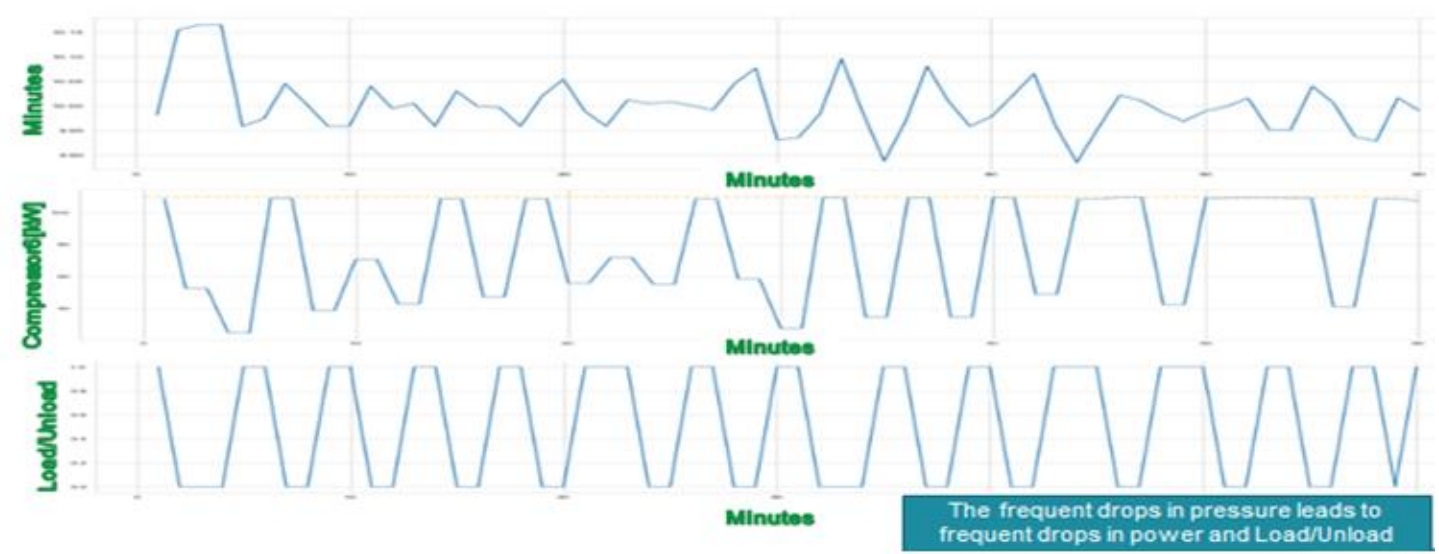

Figure 12: Shows Pressure vs Power vs Load and Unload for Day 6 Switch Count. 
Load Value Count
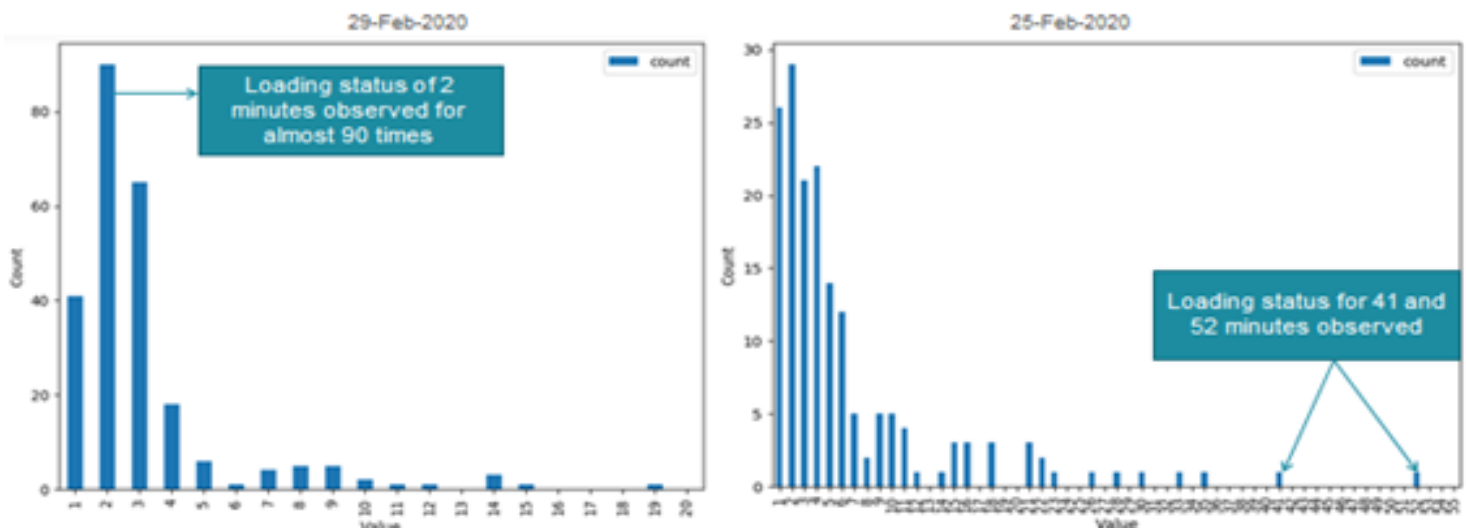

Figure 13: Shows the Load Value Count per Day.

\section{Load / Unload (Day 6)}

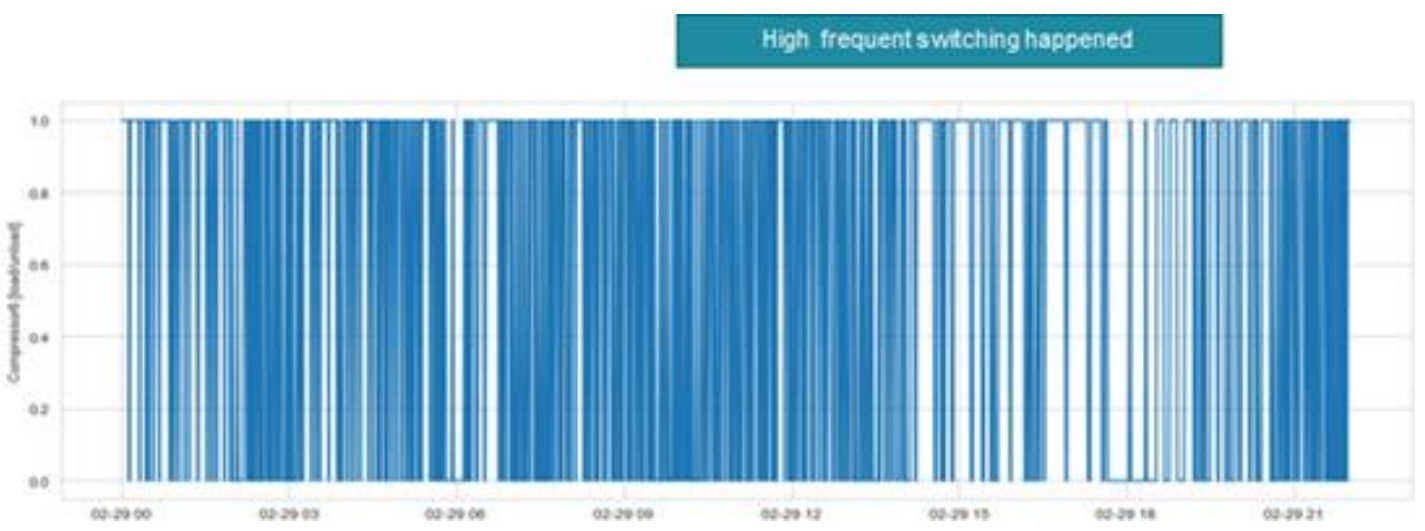

Figure 14: Shows Load and Unload for Day 6.

\section{Load / Unload (Day 2)}

Low frequent switcing happened

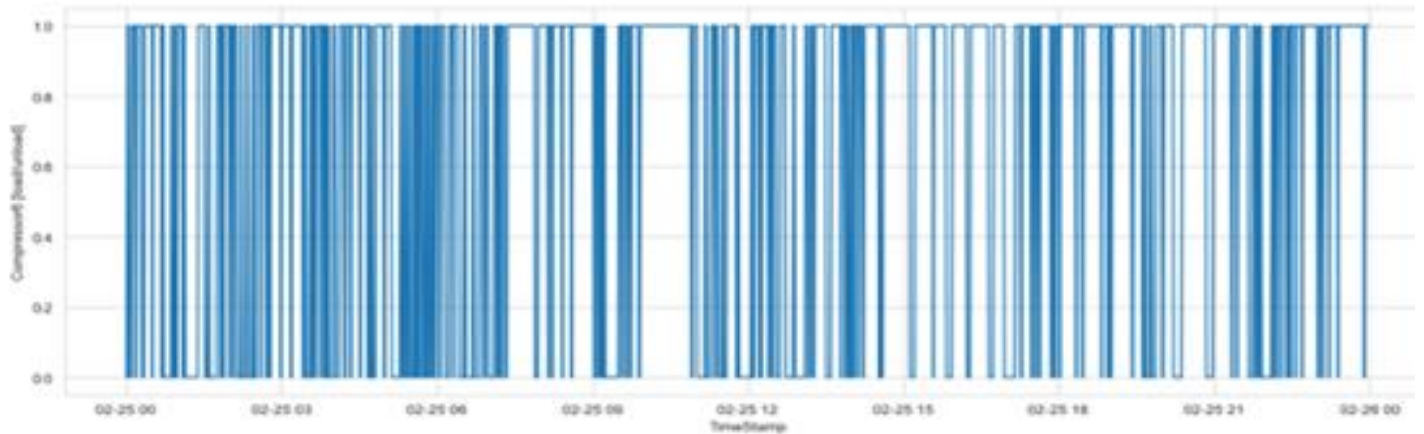

Figure 15: Shows Load and Unload for Day 2. 


\section{RESULTS AND DISCUSSIONS}

The results may be explained as follows from the graph depicted in the previous section. On the full production Day 2,Day 5 from 100 to $110 \mathrm{~kW}$ the total count is above 800 times, for the low production Day 6 the compressor has been running in sub-optimal zone and over stressed zone.

Load/unload control generates a distinctive step like power signature, drawing between $105 \%$ to $115 \%$ of rated power when loaded and between $20 \%$ and $60 \%$ of full-load power when unloaded.

Day 1 runs only half shift. For the low consumption Day 6 the frequents witching happened on the morning is high compared to afternoon.

In this way we can improve energy efficiency of all the compressor out of the six compressors and save the extra power utilized by compressors in the shopfloor.

\section{CONCLUSIONS}

Today's trend in Artificial Intelligence and machine learning has increased level of Automation in manufacturing industries allows firms to flexibly connect assets and improve productivity through data-driven insights that has not been possible before. As more automation is used in manufacturing, the speed of responses required in dealing with maintenance issues is going to get faster and automated decisions as to what's the best option from an economic standpoint are getting more complex. Here in this paper a generic dataset of a Smart manufacturing unit has been studied. A machine learning analytics has been deployed to get the predictive analysis which would be used for improving system performance and decision making. Various types of data analysis have been depicted in the result section. At the end of the result section, the prediction utilization of air comressor has been calculated which is highly satisfied.Therefore, with these results it can be concluded that predictive data analysis of a smart manufacturing system has been successfully implemented here using artificial intelligence and machine learning technique which is very crucialfor preventing machine utilization and thereby machine management

\section{REFERENCES}

1. Lee YT, Kumaraguru S, Jain S, Hatim Q, Robinson S, Helu M, et al. Aclassification scheme for smart manufacturing systems' performancemetrics. Smart Sustain Manuf Syst 2017;1(1):52-74.

2. Lalanda P, Morand D, Chollet S. Autonomic mediation middleware for smartmanufacturing. IEEE Internet Comput 2017;21(1):32-9.

3. Lade P, Ghosh R, Srinivasan S. Manufacturing analytics and industrialinternet of things. IEEE Intell Syst 2017;32(3):74-9.

4. Monostori L, Márkus A, Brussel HV, Westkämpfer E. Machine learningapproaches to manufacturing. CIRP Ann Manuf Technol1996;45(2):675-712.

5. Wuest T, Weimer D, Irgens C, Klaus DT. Machine learning in manufacturing:advantages, challenges, and applications. Prod Manuf Res 2016;4(1):23-45.

6. Zhang W, Jia MP, Zhu L, Yan X. Comprehensive overview on computationalintelligence techniques for machinery condition monitoring and faultdiagnosis. Chin J Mech Eng 2017;30(4):1-14.

7. R. N. Shaw, PratimaWalde and AnkushGhosh, "Enhancement of Power and Performance of 9x4 PV Arrays by a novel arrangement withshade dispersion", Test Engineering and Management, ISSN: 0193 - 4120 Page No. 13136-13146, 2020. 
8. R. N. Shaw, PratimaWalde and AnkushGhosh, "Effects of Solar Irradiance on Load Sharing of Integrated Photovoltaic System with IEEE Standard Bus Network”, International Journal of Engineering and Advanced Technology,Volume9 Issue-1, October 2019.

9. R. N. Shaw, PratimaWalde and AnkushGhosh, "A New Model to Enhance the Power and Performances of 4x4 PV Arrayswith Puzzle Shade Dispersion”, International Journal of InnovativeTechnology and Exploring Engineering, Volume-8 Issue-12

10. Lu C, Wang Z, Qin W, Ma J. Fault diagnosis of rotary machinery componentsusing a stacked denoising autoencoder-based health state identification.Signal Process 2017;130:377-88.

11. Chen Z, Deng S, Chen X, Li C, Sanchez RV, Qin H. Deep neural network-basedrolling bearing fault diagnosis. Microelectron Reliab2017;75:327-33.

12. Zhao R, Wang D, Yan R, Mao K, Shen F, Wang J. Machine health monitoringusing local feature-based gated recurrent unit networks. IEEE TransaIndElectron 2018;65(2):1539-48.

13. Zhao R, Yan R, Wang J, Mao K. Learning to monitor machine health withconvolution bi-directional LSTM networks. Sensors 2017; 17(273):1-18.

14. Wu Y, Yuan M, Dong S, Lin L, Liu Y. Remaining useful life estimation ofengineered systems using vanilla LSTM neural networks. Neurocomputing2017;226(5):853-60.

15. Ch.V.Phani Krishna\& K.Bhargavi, "File Translator for Multi-Dimensional Design Model - A Smart Manufacturing Approach", International Journal of Computer Science and Engineering (IJCSE), Vol. 6, Issue 5,pp. 1-4

16. Mukti Gill, Bioplastic: a Better Alternative to Plastics “, "IMPACT: International Journal of Research in Applied, Natural and Social Sciences (IMPACT: IJRANSS), Vol. 2, Issue 8, pp. 115-120

17. Niranjan Malwade, Mahesh Tavare, Akshay Kamble \& Aniruddha Kakrambe, "Smart Voting System with Face Recognition ", BEST: International Journal of Management, Information Technology and Engineering (BEST: IJMITE), Vol. 2, Issue 2, pp. $31-38$

18. Md. Ajijul Bin Zabbar \& Nafiz Ahmed Chisty, "Design \& Implementation of an Unmanned Ground Vehicle (UGV) Surveillance Robot “, International Journal of Electrical and Electronics Engineering (IJEEE), Vol. 5, Issue 6, pp. 11-20 